\title{
Localization of the magnetic equivalent of the ERN and induced oscillatory brain activity
}

\author{
Julian Keil $^{\text {a,*}}{ }^{\text {, Nathan Weisz }}{ }^{\text {a,b }}$, Isabella Paul-Jordanov ${ }^{a}$, Christian Wienbruch ${ }^{a}$ \\ "Department of Psychology, University of Konstanz, Universitaetsstrasse 10, 78457 Konstanz, P.O. Box D25, Germany \\ ${ }^{\mathrm{b}}$ Zukunftskolleg, University of Konstanz, Konstanz, Germany
}

\section{A R T I C L E I N F O}

\section{Article history:}

Received 7 October 2009

Revised 21 January 2010

Accepted 2 February 2010

Available online 10 February 2010

\begin{abstract}
A B S T R A C T
It has been found in numerous electroencephalographic (EEG) studies that a negative potential arises following an erroneous response (so-called Error-Related Negativity, ERN). This typical component of the EEG has, however, proven more difficult to identify when transferring analogous paradigms to magnetoencephalography (MEG). The aim of this study was to devise and apply a paradigm to elicit erroneous responses and using MEG to measure both the error-related evoked brain activity (mERN) as well as accompanying induced oscillatory activity. Results clearly demonstrate that it is possible to measure the mERN and to identify cortical sources associated with it. Using distributed source modeling, it is possible to identify the mERN in source space and corroborate EEG findings, with the mERN generated in the anterior cingulate cortex (ACC). This supports notions regarding the role of the ACC in error monitoring and cognitive control of motor behavior. Furthermore, changes in induced oscillatory activity were observed in the theta and beta bands. This extends previous studies, which show that evoked theta activity could underlie the generation of the ERN.
\end{abstract}

\section{Introduction}

The Error Negativity (Ne) or Error-Related Negativity (ERN) is a component of the Event-Related Potential (ERP) that can be found following errors, characterized by a frontocentral negativity and a peak at approximately 100-ms post-error (Falkenstein et al., 1991; Gehring et al., 1993). Yeung et al. (2004) noted that the term ERN is used in at least three behavioral contexts: the negativity following (1) overt response errors in choice RT tasks; (2) feedback about response accuracy; and (3) late responses in deadline RT tasks. Due to its early onset-sometimes starting a couple of milliseconds prior to the actual overt error manifestation (Yeung et al., 2004)-it is assumed that the ERN reflects the activity of an error processing mechanism, which detects already generated incorrect motor commands via a central processing path (Nieuwenhuis et al., 2001) excluding sensory or proprioceptive information as an explanation. Supposedly, an efference copy retained in the brain is used to judge the accuracy of the movement (Gehring et al., 1993).

It has been found in a number of EEG studies that the ERN is generated in the anterior cingulate cortex (ACC, Miltner, 2003) and consists of mostly radial oriented sources (Luu, 2004). Research provides converging evidence that the ACC is involved in the evaluation of performance (Yeung et al., 2004). The model of conflict

\footnotetext{
* Corresponding author. Fax: +49 7531884610.

E-mail address: julian.keil@uni-konstanz.de (J. Keil).

URL: http://www.uni-konstanz.de/obob (I. Keil).
}

monitoring by Botvinick et al. (2001) proposes a role of the ACC in attention and control in information processing possibly reflecting the psychological mechanism associated with the ERN. The cascade-ofcontrol model by Banich (2009; Banich et al., 2009) also proposes the dorsal ACC to be involved in processes related to response evaluation and selection. The ACC is regarded as a neural means of transformation of motor intentions into actions, deciding which motor action to execute from a number of possible options. Thus, if the control mechanism detects an erroneous response to an action (i.e., the chosen reaction did not lead to the desired outcome), a reduction of dopamine acts as feedback about the unsatisfying result of the performed action. This error response in turn is used as a feedback to train the ACC in order to optimize performance and action selection for the task at hand (Holroyd and Coles, 2002).

As mentioned above, proprioceptive information appears not to be directly relevant for the ERN. However, it seems to underlie a second error processing mechanism, the so-called Error Positivity (Pe). It can often be found following the ERN and possibly reflects a responseoriented P300 waveform (Falkenstein et al., 1991). The P300 is supposedly related to attention and memory updating of discrete events (Ishii et al., 2009). It has been proposed that the Pe is generated following the conscious detection of an error (Nieuwenhuis et al., 2001), the adaptation of a response strategy, or the subjective appraisal of the error (Falkenstein et al., 2000). Whereas it is assumed that the ERN is generated within the anterior cingulate cortex (ACC; Miltner et al., 2003), the Pe is supposedly generated within the posterior cingulate cortex (Wiersema et al., 2007). 
So far, only one published study (Miltner et al., 2003) was able to identify the magnetoencephalographic equivalent to the ERN ( $m E R N$ ) and to locate its generator in the ACC by means of a singledipole model. Another study (Stemmer et al., 2004) applied a flanker task during MEG and used equivalent current dipoles (ECDs) to locate the recorded activation. Although this study verified the occurrence of the ERN with a single EEG electrode during the MEG recording session, it failed to localize the generator of the MERN in the ACC.

Whereas event-related activity in response to errors has been examined extensively, oscillatory brain activity has come into focus only recently. Synchronization and desynchronization of neural oscillations might be mechanisms by which neurons within and/or between different brain regions can interact (Fries, 2005).

A number of studies (Luu et al., 2004; Cavanagh et al., 2009) have reported changes in evoked oscillatory brain activity in relation to error detection and processing mechanisms. These studies found an increase in frontal midline theta activity beginning $\sim 100 \mathrm{~ms}$ before the incorrect responses. This oscillatory brain activity as well as the ERN has been viewed as a mechanism of interaction between action monitoring brain structures.

Aside from the role of evoked theta band oscillations in the generation of the ERN and error detection networks, a recent study (Carp and Compton, 2009) reported an increase in alpha power following the commission of errors. Growing evidence implies that alpha rhythms reflect the excitatory-inhibitory balance within sensory and motor regions, with strong alpha activity indicating an inhibitory state (Klimesch, 1999; Weisz et al., 2007). Another recent study (Mazaheri et al., 2009) found that occipital and sensorimotor prestimulus alpha-band activity predicts failure to inhibit motor responses. This study again showed an increase in frontal theta activity following errors as well as an increase in alpha activity following the commission of an error. Yet, even calculating time-frequency representations on single trials, it is difficult to estimate from these studies to what extent effects reflect evoked activity (and therefore basically the same information as the ERP/ERF) or genuine, nonphase-locked, oscillatory activity. The present study tried to disentangle this issue by subtracting the evoked activity from the single trials, to examine so-called induced activity.

One goal of the present study was to measure the error-related field in the MEG and to localize the generator of the ERN using a distributed source model. Since previous studies indicate that the main ACC generator has a radial orientation, it is an unsettled issue whether the MEG-being sensitive mainly to tangentially oriented sources-can capture ERN-related activity. Compared to the EEG, the magnetic field generated in the brain and measured with the MEG is much less influenced by anatomical structures such as the cerebrospinal fluid, dura mater, skull, and skin. Thus, MEG can in principle identify neural sources more accurately than EEG (Ishii et al., 2009). In addition, magnetometers are explicitly capable of measuring signals from deep sources. Although the study by Miltner et al. (2003) lends tentative support to the notion that the mERN (as well as the ERN) are generated within the ACC, the sample size was relatively small $(N=6)$. Furthermore, only a single dipole was fitted in the individual subjects to account for the recorded brain activity. Measuring a larger sample as well as applying a distributed source model to identify the mERN could corroborate existing knowledge and more firmly establish the concept of the magnetic equivalent of the ERN. A further aim was to investigate accompanying induced oscillatory brain activity and their cortical origins. A number of studies have reported mostly evoked changes in oscillatory brain activity associated with errors, especially in the theta band. Yet the question remains which oscillatory activities are nonphase-locked to the commission of the error, thus reflecting genuinely induced oscillations.

\section{Materials and methods}

Subjects

Thirteen ( 5 males/8 females; age 23.7 years) student volunteers participated in this study as part of a course in neuropsychology. All participants gave their written informed consent according to the Declaration of Helsinki (Rickham, 1964).

\section{Experimental design and apparatus}

The participants were informed about the procedure and familiarized with the facilities. They were then prepared for the recording session and seated in the magnetically shielded room.

The stimulation consisted of 660 trials with the presentation of a computerized adaptation of the "d2 Test" (Brickenkamp, 2002), a common neuropsychological test assessing short-term attention under time pressure. Fig. 1 depicts sample trials with the timing and sequence of stimuli and response. The participants had to indicate in this choice reaction-time task as fast as possible (within $1000 \mathrm{~ms}$ ) via button press, whether the presented stimulus was a "d" accompanied by two vertical lines or not. Trials with reaction times below $280 \mathrm{~ms}$ were excluded from the offline analysis based on the assumption that these fast responses represent involuntary reactions. This cut-off value was chosen to exclude outliers based on a box plot of the reaction times (outliers were defined as data points deviating \pm 1.5 times the interquartile range from the median). Feedback was given immediately after the response. Competing stimuli were either a " $\mathrm{d}$ " or a "p" accompanied by one, three, or four marks. Stimuli were presented for $40 \mathrm{~ms}$ on a screen inside the magnetically shielded MEG acquisition room via a video projector (DLA-G11E, JVC, Friedberg, Germany) and a set of mirrors positioned outside the room.

Before the stimuli appeared on the screen, a blank screen was shown for $50 \mathrm{~ms}$. After the stimuli, feedback was given either in form of a green check mark for correct responses or a red cross for incorrect responses for $300 \mathrm{~ms}$.

\section{Data acquisition and analysis}

Magnetoencephalographic (MEG) recording was done with a 148channel magnetometer (MAGNES 2500 WH, 4D Neuroimaging, San Diego, USA). A subject-specific headframe coordinate reference was defined by means of five anatomical landmarks. These head fiducials, five coils and the subject's head shape were digitized with a Polhemus

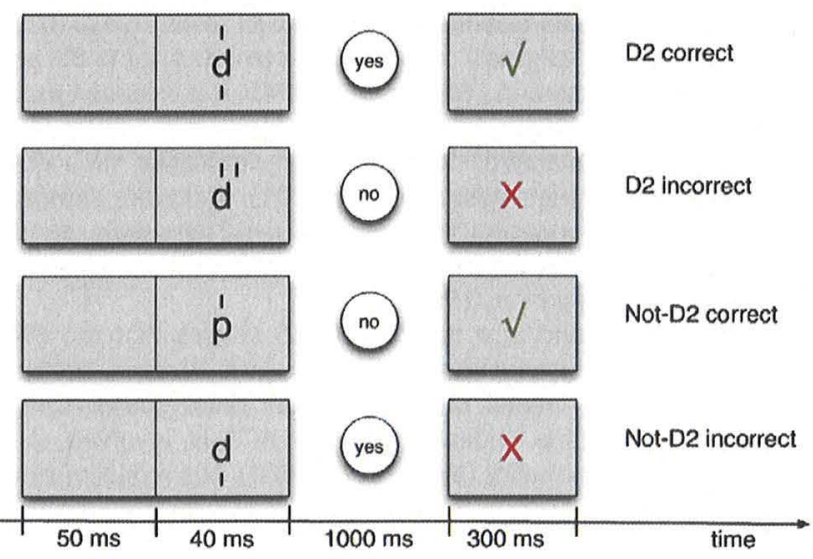

Fig. 1. The timing sequence of stimuli presented on screen as well as the four different response categories. Subjects had to indicate their choice immediately after the target presentation. Reaction times below $280 \mathrm{~ms}$ were excluded from the analysis. Reaction times above $1000 \mathrm{~ms}$ resulted in an error feedback. Feedback was given immediately after the reaction. 
3Space Fasttrack at the start of each session. The subject's head position relative to the pickup coils and the MEG sensors was estimated before and after each session to ensure that no large movements occurred during the data acquisition session.

The subjects were lying supine in a comfortable position. They were instructed to lie still during the stimulation and to avoid eye movements and blinks as much as possible. Continuous data sets were recorded with a sampling rate of $678.17 \mathrm{~Hz}$ (bandwidth $0.1-200 \mathrm{~Hz}$ ). For artifact control, eye movements (EOG) were recorded (Synamps; Neuroscan) from four electrodes attached near the left and right outer canthi and above and below the right eye. A video camera installed inside the MEG chamber allowed monitoring of the subjects' behavior and compliance throughout the experiment

After the data acquisition, epochs of $4 \mathrm{~s}( \pm 2 \mathrm{~s})$ around each button press were extracted from the raw data. The trials were visually inspected for EOG, ECG, or movement artifacts.

Trials were grouped into four different response categories ("D2 Correct", "D2 Incorrect", "Not D2 Correct", and "Not D2 Incorrect", see Fig. 1). Reaction times and relative distribution in each subject were computed from the raw trials in Matlab. Subsequently, these indices compared in order to detect different response patterns and reaction latencies. No significant differences between the two correct and the two incorrect responses were detected (see Results). In order to maximize the number of trials, the two correct and two incorrect categories were combined.

The numbers of trials with correct and incorrect responses were equalized by randomly choosing the number of trials from the smaller data set (the "incorrect" set) from the larger data set (the "correct" set) for each subject (minimum 38, maximum 118, median 75 trials). This was to ensure an identical improvement in signal-to-noise ratios for both conditions. Single trials were filtered with a $1-\mathrm{Hz}$ high-pass filter (zero-phase, Butterworth).

Artifact-free trials were then averaged and used for the analysis of event-related fields and oscillatory activity. A baseline from $100 \mathrm{~ms}$ before until onset of button press was used for baseline adjustment in the analysis of the event-related fields. As the preresponse activation was also of interest, no baseline was defined in the analysis of the oscillatory activity.

A multi-taper time-frequency transformation based on multiplication in the frequency domain using Slepian sequences as tapers was used to assess the changes in the time-frequency domain. Average event-related activity was subtracted from the single trials before computing the time-frequency transformation. This procedure resulted in single-trial estimates of oscillatory power with $\pm 0.3^{*} f$ $\mathrm{Hz}$ frequency smoothing. The investigated frequency band ranged from $2 \mathrm{~Hz}$ to $70 \mathrm{~Hz}$ in logarithmic steps.

The individual head shapes collected prior to the actual experiment were used to generate pseudo-individual MRI images. Here, the canonical cortical anatomy was obtained automatically from the affine transformation of an MNI-template brain to the subject's digitized head shape (Lecaignard et al., 2008). Source activities were projected onto these approximate individual anatomical MRI images and subsequently normalized onto a standard MNI brain (Montreal Neurological Institute (MNI), Montreal, Canada; http://www.bic.mni. mcgill.ca/brainweb) using SPM2 (http://www.fil.ion.ucl.ac.uk/spm), in order to accomplish group statistics and for illustrative purposes.

A linear constrained minimum variance beamformer algorithm (LCMV, Van Veen et al., 1997) was used to identify the sources of the effects found in the time-series analysis. Source analysis was performed separately for a baseline interval $(-100 \mathrm{~ms}$ before until onset of button press) and an activation interval ( $75 \mathrm{~ms}$ until $200 \mathrm{~ms}$ after button press; see Results). The source analysis was done on the waveforms of the two conditions and difference was only computed in the statistical analysis.

Dynamic imaging of coherent sources (DICS, Gross et al., 2001), a frequency-domain adaptive spatial filtering algorithm, was used to identify the sources of the effects found in the time-frequency domain. This algorithm has proven to be particularly powerful when localizing oscillatory sources (Liljeström et al., 2005). As the preresponse activation was of interest here as well, no baseline was defined and source analysis outputs for both conditions were compared directly.

In order to define relevant time and frequency windows, a clusterbased (at least 2 sensors/cluster) dependent-samples $t$-test with Monte Carlo randomization was performed on the sensor data (1000 draws for the ERF; 500 draws for the time-frequency data). This method allows the identification of clusters of significant differences in 2D and 3D (time, frequency, and space). It effectively controls for multiple comparisons (for details, see Maris and Oostenveld, 2007). Clusters were defined as significant if the probability of observing larger effects from shuffled data was below $5 \%$. The cluster-level test statistic is defined as the sum of the $t$-statistics of the sensors in the respective cluster.

In order to scrutinize the probable neuronal generators of the observed sensor effects, statistical comparisons on the source level were computed using dependent-samples t-tests.

At the suggestion of a reviewer, individual error rates (number of incorrect trials divided by the number of correct trials, i.e., high values indicate high number of errors) were correlated with the individual differences (cortical activity for the correct trials versus incorrect trials) on the source level for the time and time-frequency analyses.

All aspects of offline treatment of the MEG signals were accomplished using fieldtrip, an open-source signal processing toolbox for Matlab (Donders Institute for Brain, Cognition and Behaviour, Radboud University Nijmegen, The Netherlands. See http://www.ru.nl/neuroimaging/fieldtrip). Anatomical structures corresponding to the statistical effects were found using the MNI brain and Talairach atlas (MRC Cognition and Brain Sciences Unit, Cambridge, England. See http://imaging.mrc-cbu.cam.ac.uk/imaging/ MniTalairach).

\section{Results}

Participants performed the majority of the 660 trials correctly. Table 1 shows the average percent distribution in the different categories as well as mean reaction times. Reaction times and average percent distribution between the different categories were compared. Whereas number of errors and correct responses differed significantly $\left(\chi^{2}(36)=213.56, p<0.001\right)$, reaction times for the four categories ("D2 Correct", "D2 Incorrect", "Not D2 Correct", and "Not D2 Incorrect", see Fig. 1$)$ were equal $(F(3,48)=2.40, p=0.1, \varepsilon=0.47)$. No significant reaction time interactions between stimulus type ( $d 2$ vs. not $\mathrm{d} 2$ ) and correctness (correct vs. incorrect response) were observed. Thus, as the respective correct and incorrect trials were combined, the subjects on the average responded correct more often $\left(\chi^{2}(12)=254.11, p<0.001\right)$, but the reaction times did not differ between correct and incorrect responses $(t(25)=-1.49, p=0.14)$.

As a first step in the analysis of MEG data, event-related fields around the motor responses were analyzed so as to identify the magnetic equivalent to the ERN and its localization. In a second step, oscillatory activity in these trials was assessed via time-frequency analysis and the localization of the respective generators. Contrasts

Table 1

Average distribution and mean reaction times for the four response categories as well as correct and incorrect responses combined.

\begin{tabular}{ccccccc}
\hline & $\begin{array}{l}\text { D2 } \\
\text { correct }\end{array}$ & $\begin{array}{l}\text { D2 } \\
\text { incorrect }\end{array}$ & $\begin{array}{l}\text { Not D2 } \\
\text { correct }\end{array}$ & $\begin{array}{l}\text { Not D2 } \\
\text { incorrect }\end{array}$ & $\begin{array}{l}\text { Combined } \\
\text { correct }\end{array}$ & $\begin{array}{l}\text { Combined } \\
\text { incorrect }\end{array}$ \\
\hline $\begin{array}{c}\text { Percent of } \\
\text { trials }\end{array}$ & 80.5 & 7.2 & 78 & 6 & 79.4 & 6.7 \\
RT (ms) & 544.71 & 547.04 & 550.15 & 613.90 & 547.44 & 580.47 \\
\hline
\end{tabular}


a

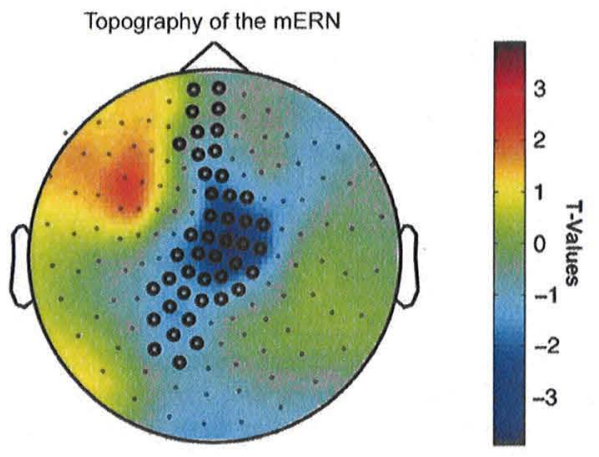

b

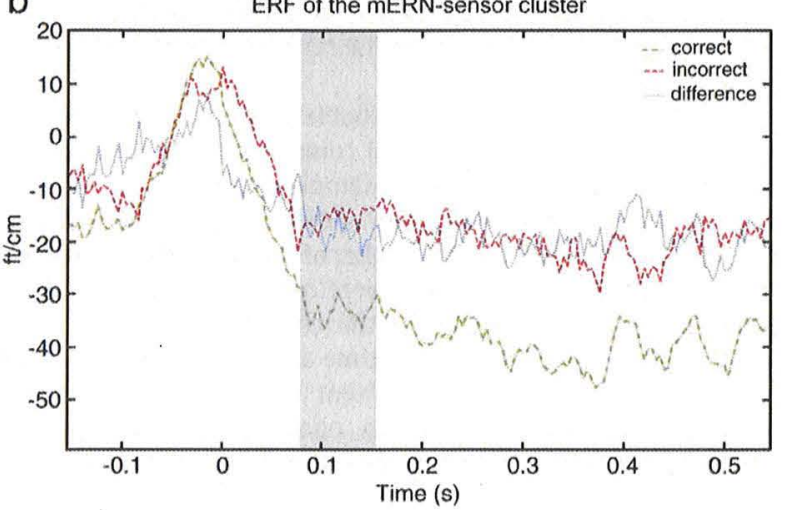

Fig. 2. (a) The topography ( $t$-values, correct vs, incorrect trials) and the negative sensor cluster found in the statistical analysis for the mERN latency (80-160 ms) and (b) the ERF trace of the negative sensor cluster for the correct (green) and incorrect (red) trials as well as the difference between the two trial types (blue; grand average over the sensors in the cluster). The mERN interval is shaded blue, button press occurred at $0 \mathrm{~ms}$.

between the correct and incorrect trials were computed on all levels of analysis.

\section{Event-related fields}

ERF analysis revealed a differential activity following correct and incorrect button presses (Fig. 2). The amplitude following incorrect responses was significantly less pronounced between $80 \mathrm{~ms}$ and 160 $\mathrm{ms}(p<0.05)$ in a sensor cluster extending from frontal to parietal sensors.

The source analysis via the LCMV beamformer suggests the left ACC to be the source of this difference (MNI coordinates [-5.0 28.0
29.0], see Fig. 3). No correlation between error rates (number of incorrect trials divided by the number of correct trials) and ACC activity was found. For individual source localizations of this effects, see Supplementary data.

Whereas a significant difference was detectable in an early time window (comprising the ERN), no differences were detected at later latencies such as the time window usually comprising the Pe.

\section{Oscillatory activity}

Time-frequency analysis revealed two clusters-one positive (i.e., greater power in the correct trials) and one negative (i.e., greater power in the incorrect trials)-of oscillatory activity significantly different between the correct and incorrect trials.

First, from $-200 \mathrm{~ms}$ before the motor response to $700 \mathrm{~ms}$ after the motor response, the incorrect trials produced greater theta-band $(2-8 \mathrm{~Hz})$ power $(p<0.05$, see Fig. 4$)$.

Cluster analysis revealed a bilateral medial-frontal sensor group in which this difference reached significance. DICS suggests that multiple frontal regions are involved in generating the difference seen at the sensor level, among others the bilateral medial frontal gyrus (MNI coordinates [35.0 46.0 21.0], see Fig. 5). A high negative correlation between error rates (number of incorrect trials divided by the number of correct trials) and theta-band activity was observed in the left medial frontal gyrus $(r \sim-0.77)$ (see Fig. 6).

Second, starting at the response and lasting until $500 \mathrm{~ms}$ thereafter (with a maximum between $100 \mathrm{~ms}$ and $400 \mathrm{~ms}$ after the response), there was greater beta-band $(15-25 \mathrm{~Hz})$ power in the correct trials ( $p<0.05$, see Fig. 7$)$ with a focus at right-parietal sensors.

Cluster analysis revealed a parietal sensor group in which this difference reached significance. DICS suggests a right-lateralized parietal cortical source including the precuneus as the source of this difference (MNI coordinates [43.0 - 73.0 40.0], see Fig. 8). A high positive correlation between error rates (number of incorrect trials divided by the number of correct trials) and beta-band activity was observed in the precuneus ( $r \sim 0.81$ ) (see Fig. 9).

\section{Discussion}

In the present MEG study, a computerized equivalent to the " $\mathrm{d} 2$ test" of attention was devised in order to provoke errors and errorrelated brain activity. The behavioral context fits the circumstances mentioned by Yeung et al. (2004) who state that the ERN can be found following overt response errors in choice reaction time tasks (in our case the erroneous reaction), following feedback about response accuracy (in our case the feedback following the reaction) and following late responses in deadline reaction time tasks (in our case the feedback following a late responses). Whereas it can be argued that the cortical activity reported here arises from a mixture of the
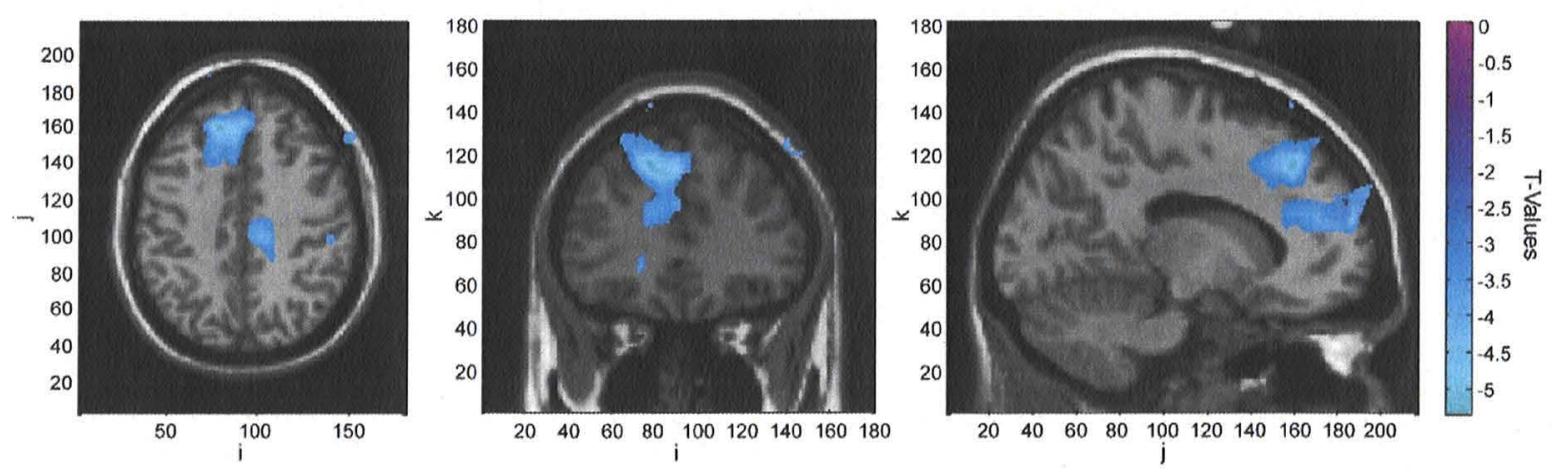

Fig. 3. The source ( $t$-values, correct vs. incorrect trials) of the effect found in the ERF analysis based on the LCMV beamformer analysis (interval from 75 ms to $200 \mathrm{~ms}$ after the response). Source analysis was performed for the correct and incorrect trials separately. 


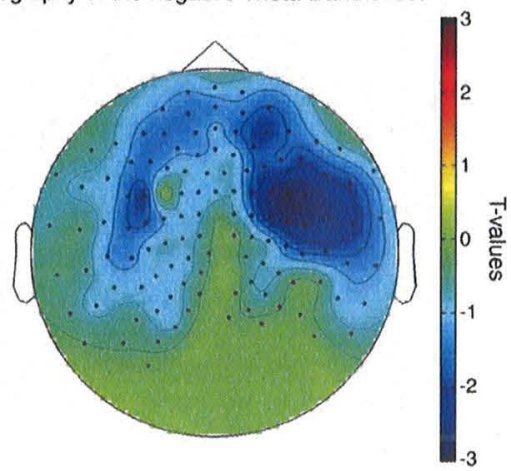

b Time-frequency representation of the negative Theta-Band effect

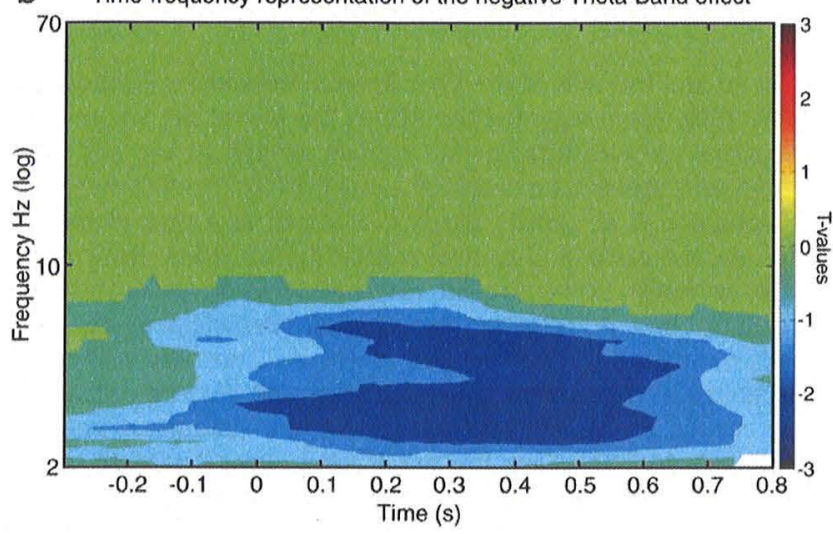

Fig. 4. The topography ( $t$-values, correct vs. incorrect trials) of the negative sensor cluster found in time-frequency analysis and the result of the time-frequency analysis (mean over sensors in the cluster, $t$-values). Button press occurred at time 0 .

processes mentioned above, we could not differentiate between them due to the immediacy of feedback in the present experiment. In this way, it is likely, that the activity measured here does not constitute a pure response-locked ERN but also feedback processing in terms of the feedback-related negativity (FRN, Miltner et al., 1997).

Whereas error-related brain activity has been widely examined in the EEG, studies using MEG are scarce. Only a few studies (Miltner et al., 2003; Stemmer et al., 2004; Mazaheri et al., 2009) investigated the magnetic equivalent of the ERN in the MEG. Whereas Miltner et al. (2003) confirmed the location of the generator of the ERN in the ACC using single-dipole modeling in the individual head volumes, Stemmer et al. (2004) failed to localize the generator of the mERN. Mazaheri et al. (2009), on the other hand, attributed a major part of the mERN activity recorded to theta activity, but from the analysis it is not possible to decipher to what extent this effect is due to processes strictly phase locked to the button press or whether they reflect induced processes. Furthermore these authors refrained from explicitly localizing the ERF data.

We hypothesized to find a differentiating magnetic response equivalent to the ERN and to localize this $\mathrm{MERN}$ in the ACC. In addition, we were interested in whether the mERN would be accompanied by changes in induced theta-band activity, a question that cannot be answered definitely based on current available studies. In order to accomplish this, averaged event-related activity was subtracted from the single trials prior to computing the time-frequency analysis.

We discuss the present results with respect to response errors. It would be also possible to analyze the present data in relation to the feedback-related activity. However, because the subjects' response triggered the feedback display, it is not readily possible to disentangle error-related processes from feedback-related processes in this study. Still, similar results can be expected, as the underlying process-the ERN-can be found in both processes. Miltner et al. (1997) described the feedback-related negativity (FRN), a negative ERP component that corresponds to the ERN in many aspects.

\section{Behavioral results}

In the analysis of the error rates and reaction times of the different response categories, it became apparent that although the experimental task was designed to increase the error rate, the majority of the trials were performed correctly. Whereas it can be argued that the two different error conditions constitute distinct response categories (i.e., missing a response vs. producing a false positive response) and thus represent different types of response conflict, it has been shown (Yeung et al., 2004) that both reactions produce some common patterns of cortical activation, which is emphasized by averaging over both error types. In order to ensure a large enough number of trials for meaningful statistical comparisons and on the basis that no differences were found between the different trial categories with respect to reaction times, the correct and incorrect categories were grouped together. Future studies could be specifically designed to further increase the error rate and thus make it possible to contrast different types of response conflict.

Although a neuropsychological standard instrument was adapted for the MEG, the results obtained in this experiment are not readily comparable to results obtained with the original test. Whereas a line of stimuli is presented to work on in the original test, stimuli were displayed in fast succession with immediate feedback. Together with the circumstances of the recording session inside the MEG chamber, the high time pressure due to the speeded presentation of stimuli could be the reason that no post-error slowing or significant differences between the response categories were found.

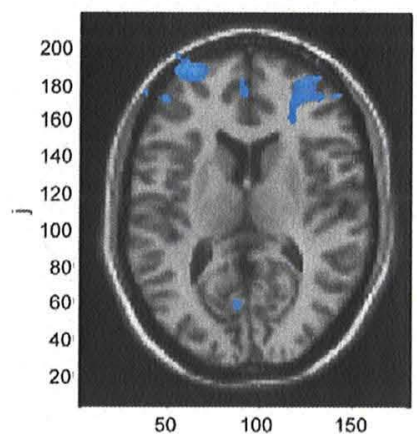

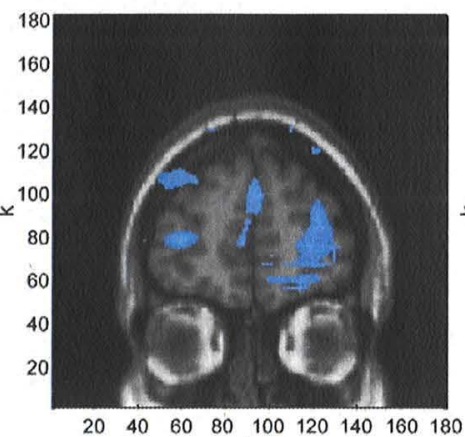

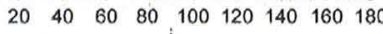

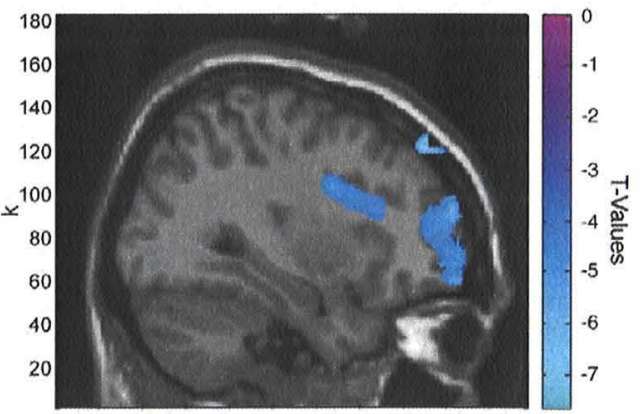

$\begin{array}{llllllllll}20 & 40 & 60 & 80 & 100 & 120 & 140 & 160 & 180 & 200\end{array}$

Fig. 5. The source ( $t$-values, correct vs. incorrect trials) of the effect found in the theta band based on the DICS beamformer analysis (frequency range from $2 \mathrm{~Hz}$ to $8 \mathrm{~Hz}$, time interval from - $200 \mathrm{~ms}$ before the response to $700 \mathrm{~ms}$ thereafter). Source analysis was performed for the correct and incorrect trials separately. 

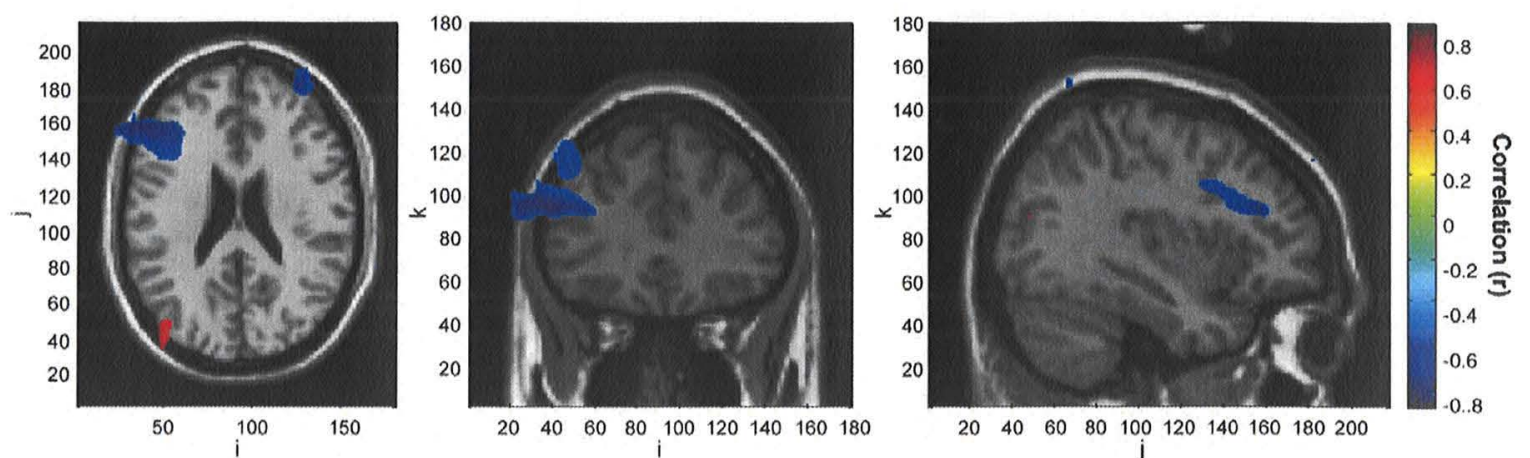

Fig. 6. Correlations between the individual source projections of the theta-band effect and the respective error rates. A negative correlation indicates that low error rates were correlated with high theta-band activity.

\section{Event-related potentials}

In line with the first hypothesis, the present study affirmed that it is possible to measure error-related activity in the MEG and to identify its generator. In accordance with the studies mentioned above, we measured a differential activity in a period from $80 \mathrm{~ms}$ to $160 \mathrm{~ms}$ following an incorrect response. This is the time interval given by the majority of research articles reporting on the ERN. Interestingly though, a slight delay in the onset of the mERN was reported by Stemmer et al. (2004) as well as by Mazaheri et al. (2009). They reported the latency of the $\mathrm{mERN}$ to be approximately between $70 \mathrm{~ms}$ and $190 \mathrm{~ms}$. Although the onset of the ERN varies in the EEG, this delay was attributed by both sets of authors to the measurement of different neuronal networks in EEG and MEG.

In the EEG literature, the ERN is described as a negative potential with a frontocentral topography in the EEG (Falkenstein et al., 1991; Gehring et al., 1993) resulting from a radial-oriented ACC source (Luu, 2004). At a first glance this seems to be contradictory to the more negative deflection for correct trials, which we identified using a cluster-based statistical approach. Even though it is tempting to directly relate our reported topography to EEG results, this cannot be done due to several reasons: First of all both methods measure different aspects following (synchronous) post-synaptic activity in large neuronal ensembles. Whereas the EEG signal reflects extracellular volume currents, signals measured by the MEG are putatively generated by intracellular currents (Baillet et al., 2001). Furthermore as mentioned above the dominant EEG generator is reported to be radial, perhaps obscuring contributions of tangential sources. However MEG is mostly sensitive to activity from tangentially oriented sources. The topography of the statistical map in Fig. 2a indeed suggests a tangential source: besides of the negative frontocentral cluster, there is a left frontal cluster with positive values (meaning stronger negativity for incorrect responses). Our nonparametric cluster-based permutation test only identified the negative cluster as significant. If our statistical map in Fig. 2a would reflect the difference to come from a single source, then in an ideal case, the positive cluster should have been equally significant. It is possible that MEG sensors at left frontal sites capture activity from other sources as well, therefore obscuring an effect for the positive cluster. Overall, aspects of topography and polarity cannot be transferred directly between EEG and MEG, and both methods may contribute complimentary information also regarding error processing.

Nevertheless, we are still confident that the evoked effect identified using MEG still reflects an ERN-like activity. Firstly, the contrast between correct and incorrect responses has a similar time frame as reported in the EEG literature. Moreover, we localized the mERN in the ACC with a left-hemispheric lateralization. This corroborates the role of the ACC in error monitoring and cognitive control of motor behavior and lends credence to theories of ACC function and the role of the ERN. Several researchers (Holroyd and Coles, 2002) have regarded the ACC as the neural site where motor intentions are transformed into actions as well as the site where erroneous responses are detected. In the model of conflict monitoring by Botvinick et al. (2001) the ACC is stated as a core element. In addition, the cascade-of-control model (Banich, 2009; Banich et al., 2009) attributes a central role in response selection and evaluation to the ACC. Thus, in a comparison process (Bernstein et al., 1995), conflict monitoring implemented in the ACC possibly reflects this psychological mechanism (i.e., comparing and detecting unsatisfactory results of an act and subsequent modulation of behavior) associated with the ERN, the psychophysiological marker of error detection or error compensation. Miltner et al. (1997) describe an ERP component following negative feedback that corresponds to the ERN.

a

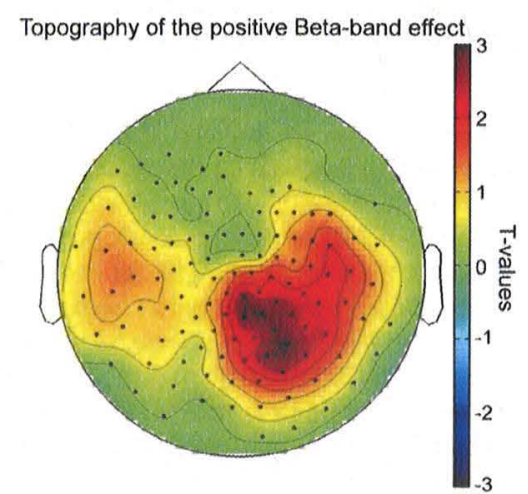

b

b Time-frequency representation of the positive Beta-band effect

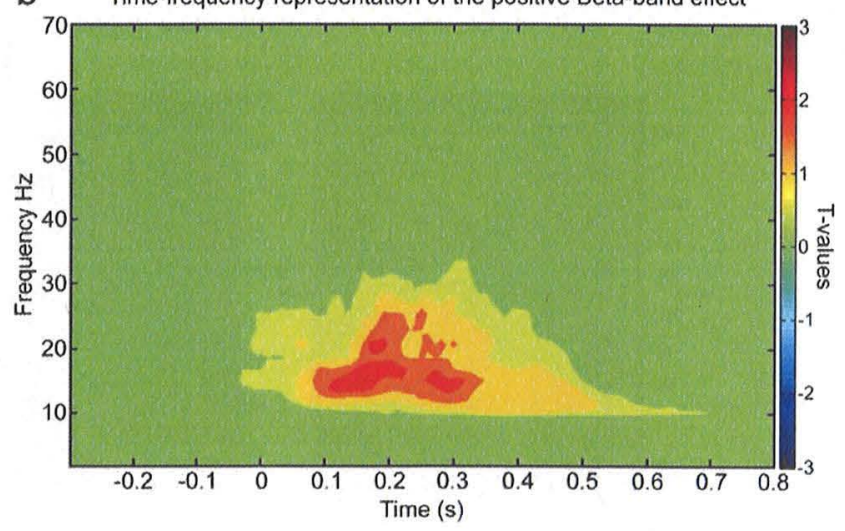

Fig. 7. The topography ( $t$-values, correct vs. incorrect trials) of the positive sensor cluster found in the time-frequency analysis and the result of the time-frequency analysis (mean over sensors in the cluster, $t$-values). Button press occurred at time 0. 


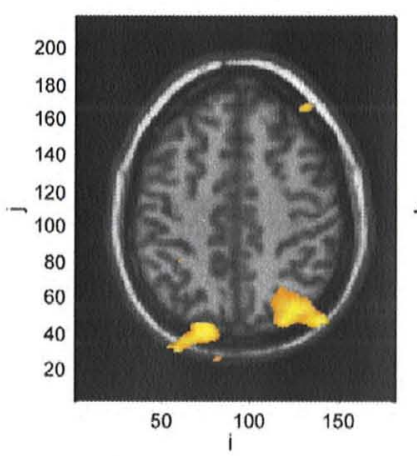

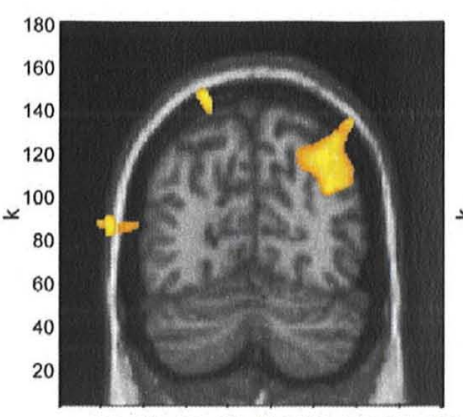

$20 \quad 40 \quad 60 \quad 80 \quad 100120140160180$

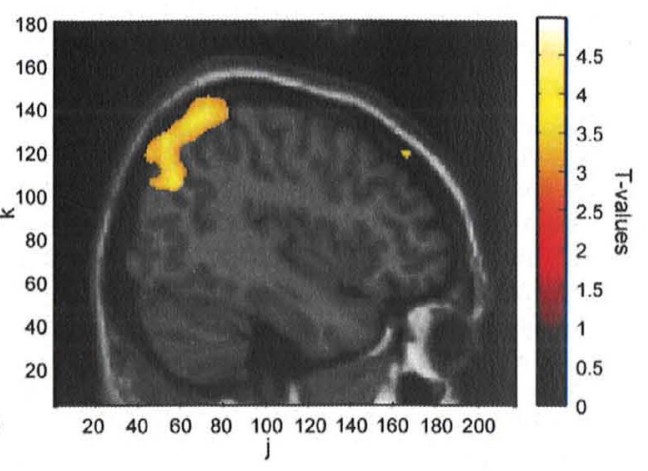

$20 \quad 40 \quad 6080100120140160180200$

Fig. 8. The source ( $t$-values, correct vs. incorrect trials) of the effect found in the beta band based on the DICS beamformer analysis (frequency range from $15 \mathrm{~Hz}$ to $25 \mathrm{~Hz}$, time interval from $100 \mathrm{~ms}$ to $400 \mathrm{~ms}$ after the response). Source analysis was performed for the correct and incorrect trials separately.

Although we cannot disentangle error-related processes from feedback-related processes in the present study due to the immediacy of feedback, these results could be attributed to feedback-related negativity. This would lend support to Miltner's notion of a generic error detection mechanism that can be adjusted to the current task and goals. Whereas a significant difference following correct and incorrect reactions both at the sensor level and at the source level could be measured in the ERN interval, no difference was detected in the Pe interval. This could be due to the likely deeper location of the dipole(s) in the cortical areas generating the respective processes, which makes the Pe readily detectable in the EEG but not in the MEG.

\section{Induced oscillatory processes}

In line with the second hypothesis, we analyzed oscillatory activity. We found an increase in induced theta-band power following the incorrect trials from $-200 \mathrm{~ms}$ before the motor response until $700 \mathrm{~ms}$ after the response. In the time interval from $100 \mathrm{~ms}$ to $400 \mathrm{~ms}$ after the response, we found an increase in induced beta-band activity in the correct trials.

Oscillatory brain activity has been reported to play a role in the generation of the ERN (Trujullo and Allen, 2007) as well as in relation to error detection and processing mechanisms (Luu et al., 2004; Cavanagh et al., 2009). Increases in frontal midline phase-locked theta oscillations have been proposed to be responsible for the generation of the ERN. Our findings partly match these results in timing and location, but source analysis implies a different generator for the induced theta activity than for the mERN. It is important to note that the induced theta activity is independent from event-related activity. Induced theta oscillations appear to emerge from bilateral medial frontal gyrus, not from ACC as for the evoked data. However, the time interval as well as the location of the increase in theta power at both the sensor and source levels overlaps with the activity ascribed to the mERN. This supports the link between induced theta oscillations and processing of errors and also underscores that not only event-related but also ongoing changes play an important role in error processing. It suggests that relevant information is lost when averaging time-series data, since this process penalizes nonphase-locked activities as reported here. The time interval reported here is-due to the analyzed frequency spectrum-relatively large. That means that both automatic error processing as represented by the ERN and conscious error processing as represented by the Pe occur in this time interval. Still, we refrain from associating the activity measured here to the Pe. First, no evidence for the Pe was found in the analysis of the event-related fields, which might be due to the orientation and depth of location of dipoles. Second, the source analysis points to frontal generators for this activity, which is more in line with the evidence of the ERN being generated in frontal cortex. It is important to note that the differences in source localization between the mERN and the induced theta-band activity could reflect different aspects of error processing. Whereas the ERN is supposed to consist in part of evoked theta oscillations, with an ACC generator (Luu, 2004) the induced theta oscillations observed here might represent changes in the ongoing activity of a prefrontal performance-monitoring network, as proposed by Miltner in the generic system for error detection (Miltner et al., 1997). As the changes in induced theta activity already occur before the actual response, these gradual modulations might lead to errors in task performance (i.e., an increase in error rate) or changes in the ability to detect errors due to a maladaptive state of the performancemonitoring system.

Whereas changes in the theta band have been linked to error processing mechanisms, beta-band activity has been linked most prominently to attention and awareness processes (Fan et al., 2007). In this study, we found an increase of beta-band activity following
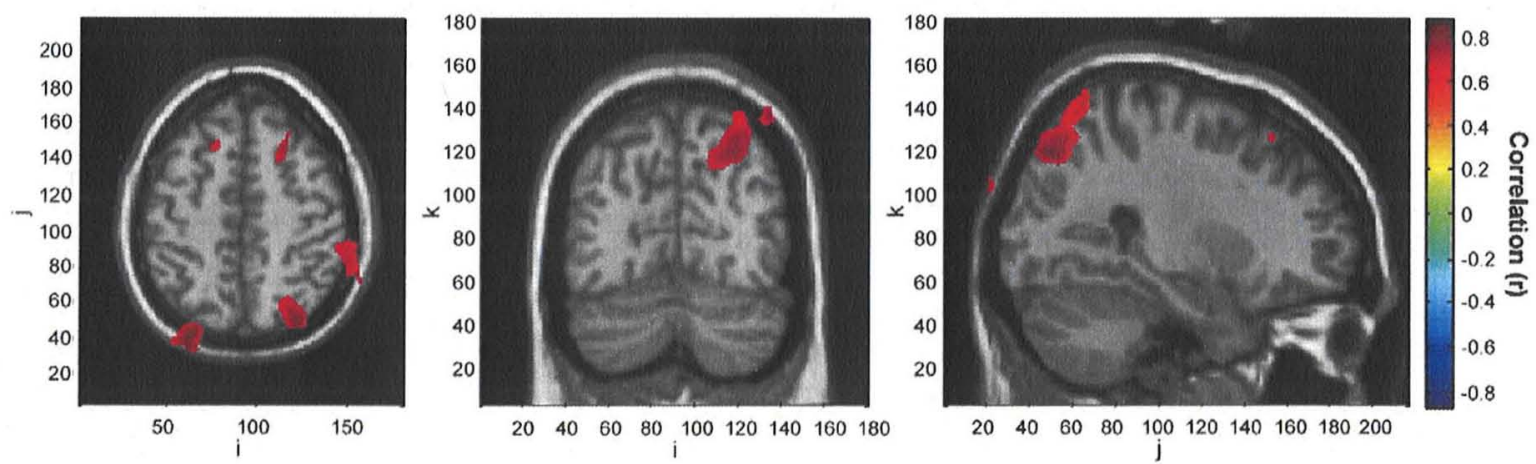

Fig. 9. Correlations between the individual source projections of the beta-band effect and the respective error rates. A positive correlation indicates that high error rates were correlated with high beta-band activity. 
correct trials with an occipital-parietal topography. The beamforming source analysis identified a parietal cluster of activation, including the precuneus, to be the source of this increase in activity. As beta-band activity is possibly linked to cognitive processes as well as emotion, the increase following the correct trials could point to an increased processing of the correct trials, as they are motivationally important to the subject and the behavior. The precuneus has been linked to error processing in fMRI studies by Menon et al. (2001) and Eichele et al. (2008). Whether the relative beta-band increase following the correct responses found in this study is related to the Pe is unclear, as no evidence for a magnetic equivalent of the Pe was found in the analysis of the ERF data. The fMRI study by Eichele et al. (2008) suggested gradual changes in the default-mode network containing the precuneus to be responsible for a substantial proportion of errors in a flanker task.

The individual error rate is highly correlated with the effects found in the theta band ( $r \sim-0.77$ in medial frontal gyrus) and beta band $(r \sim 0.81$ in the precuneus), although no correlation was found between error rate and mERN amplitude. These results suggest a gradual relationship between behavior (i.e., effective performance monitoring and attention reflected by few errors) and oscillatory activity in the theta and beta bands. High error rates were correlated with low theta-band activity, which could indicate ineffective performance monitoring. Based on the latency of the induced theta activity (an increase $\sim 200 \mathrm{~ms}$ before the incorrect response), it could also be possible that the correlation with error rate indicates an errortripping role of theta oscillations. In turn, the ERN, generated in the ACC, could act as an error detection mechanism. In this way, it would be plausible why induced theta activity but not the mERN correlates with performance. High error rates were correlated with high betaband activity, which could indicate relatively low error awareness and attention to incorrect trials but a focus on correct trials. This adds evidence to the importance of the changes in ongoing brain oscillations for behavioral performance and underscores the functional relevance of the effects found in this study. Moreover, these results could lend support to the notion of a generic error detection mechanism (Miltner et al., 1997), as gradual changes in the cortical areas described here are closely linked to performance.

To summarize, our results corroborate the concept of an mERN (Miltner, 2003) and also support the notion of the ACC as the generator of the ERN. The complementary information gained from EEG and MEG could be used in future studies to elucidate functional differences between radial and tangential sources. Moreover, the role of theta oscillations in error processing was extended by demonstrating the presence of induced activity, which, however, is not generated in the ACC but bilaterally in the medial prefrontal gyrus. In addition, the presence of induced beta-band activity with a source in the precuneus was established, suggesting modulations in attention and motivation associated with the processing of errors.

\section{Acknowledgments}

J.K. and N.W. are supported by grants from the Deutsche Forschungsgemeinschaft (DFG; WE 4156/2-1). The authors wish to thank Ursula Lommen for help with data acquisition and management, Jérémie Mattout and Françoise Lecaignard for their support and Matlab code in order to generate the pseudo-individual MRIs used in the present study, and two reviewers for their very helpful and constructive comments.

\section{Appendix A. Supplementary data}

Supplementary data associated with this article can be found, in the online version, at doi:10.1016/j.neuroimage.2010.02.003.

\section{References}

Baillet, S., Mosher, J., Leahy, R., 2001. Electromagnetic brain mapping. IEEE Signal Process. Mag. 18 (6), 14-30.

Banich, M., 2009. Executive function: the search for an integrated account. Curr. Dir. Psychol. Sci. 18 (2), 89-94.

Banich, M., Mackiewicz, K., Depue, B., Whitmer, A., Miller, G., Heller, W., 2009. Cognitive control mechanisms, emotion and memory: a neural perspective with implication for psychopathology. Neurosci. Biobehav. Rev. 33 (5), 613-630.

Bernstein, P., Scheffers, M., Coles, M., 1995. "Where did I go wrong?" A psychophysiological analysis of error detection. J. Exp. Psychol. Hum. Percept. Perform. 21, 1312-1322.

Botvinick, M., Braver, T., Barch, D., Carter, C., Cohen, J., 2001. Conflict monitoring and cognitive control. Psychol. Rev. 108 (3), 624-652.

Brickenkamp, R. (2002). Test d 2 Aufmerksamkeits-Belastungs-Test. Hogrefe, Göttingen (9).

Carp, Compton, R, 2009. Alpha power is influenced by performance errors. Psychophysiology 46 (2), 336-343.

Cavanagh, J., Cohen, M., Allen, J., 2009. Prelude to and resolution of an error: EEG phase synchrony reveals cognitive control dynamics during action monitoring. J. Neurosci. 29 (1), 98-105.

Eichele, T., Debener, S., Calhoun, V., Specht, K., Engel, A., Hugdahl, K., et al., 2008. Prediction of human errors by maladaptive changes in event-related brain networks. Proc. Natl. Acad. Sci. U. S. A. 105 (16), 6173-6178.

Falkenstein, M., Hohnsbein, J., Hoormann, J., Blanke, L., 1991. Effects of crossmoda divided attention on late ERP components: II. Error processing in choice reaction tasks. Electroencephalogr. Clin. Neurophysiol. 78 (6), 447-455

Falkenstein, M., Hoormann, J., Christ, S., Hohnsbein, J., 2000. ERP components on reaction errors and their functional significance: a tutorial. Biol. Psychol. 51 (2-3), 87-107.

Fan, J., Byrne, J., Worden, M., Guise, K., Mccandliss, B., Fossella, J., et al., 2007. The relation of brain oscillations to attentional networks. J. Neurosci. 27 (23), 6197-6206.

Fries, P., 2005. A mechanism for cognitive dynamics: neuronal communication through neuronal coherence. Trends Cogn. Sci. 9 (10), 474-480.

Gehring, W., Goss, B., Coles, M., Meyer, D., Donchin, E., 1993. A neural system for error detection and compensation. Psychol. Sci. 4 (6), 385-390.

Gross, J., Kujala, J., Hamalainen, M., Timmermann, L., Schnitzler, A., Salmelin, R., 2001. Dynamic imaging of coherent sources: studying neural interactions in the human brain. Proc. Natl. Acad. Sci. U. S. A. 98 (2), 694-699.

Holroyd, C., Coles, M., 2002. The neural basis of human error processing: reinforcement learning, dopamine, and the error-related negativity. Psychol. Rev. 109 (4), 679-709.

Ishii, R., Canuet, L., Herdman, A., Gunji, A., Iwase, M., Takahashi, H., et al., 12. Jan 2009 Cortical oscillatory power changes during auditory oddball task revealed by spatially filtered magnetoencephalography. Clin. Neurophysiol. 8

Klimsch, W., 1999. EEG alpha and theta oscillations reflect cognitive and memory performance: a review and analysis. Brain Res. Rev. 29, 169-195.

Lecaignard, F., Bouet, R., Mattout, J., 8. Aug 2008. Comparing models of cortical anatomy for MEG source reconstruction. Biomag. 2008.

Liljeström, M., Kujala, J., Jensen, O., Salmelin, R., 2005. Neuromagnetic localization of rhythmic activity in the human brain: a comparison of three methods. Neurolmage 25 (3), 734-745.

Luu, P., Tucker, D., Makeig, S., 2004. Frontal midline theta and the error-related negativity: neurophysiological mechanisms of action regulation. Clin. Neurophysiol. 115 (8), $1821-1835$.

Maris, E., Oostenveld, R., 2007. Nonparametric statistical testing of EEG- and MEG-data. J. Neurosci. Methods 164 (1), 177-190.

Mazaheri, A., Nieuwenhuis, I., Van Dijk, H., Jensen, O., 2009. Prestimulus alpha and mu activity predicts failure to inhibit motor responses. Hum. Brain Mapp. 30 (6) $1791-1800$.

Menon, V., Adleman, N., White, C., Glover, G., Reiss, A., 2001. Error-related brain activation during a Go/NoGo response inhibition task. Hum. Brain Mapp. 12 (3) $131-143$.

Miltner, W. Braun, H., Coles, M., 1997. Event-related brain potentials following incorrect feedback in a time-estimation task: evidence for a "generic" neural system for error detection. J. Cogn. Neurosci. 9 (6), 788-798.

Miltner, W., 2003. Implementation of error-processing in the human anterior cingulate cortex: a source analysis of the magnetic equivalent of the error-related negativity. Biol. Psychol. 64 (1-2), 157-166.

Nieuwenhuis, S, Ridderinkhof, K., Blom, J., Band, G., Kok, A, 2001, Error-related brain potentials are differentially related to awareness of response errors: evidence from an antisaccade task. Psychophysiology 38 (5), 752-760.

Rickham, P., 1964. Human experimentation. Code of ethics of the world medical association. Declaration of Helsinki. British Med. J. 2 (5402), 177.

Stemmer, B., Vihla, M., Salmelin, R., 2004. Activation of the human sensorimotor cortex during error-related processing: a magnetoencephalography study. Neurosci. Lett. 362 (1), 44-47.

Trujillo, L., Allen, J., 2007. Theta EEG dynamics of the error-related negativity. Clin. Neurophysiol. 118 (3), 645-668

Van Veen, B., van Drongelen, W., Yuchtman, M., Suzuki, A., 1997. Localization of brain electrical activity via linearly constrained minimum variance spatial filtering. IEEE Trans. Biomed. Eng. 44 (9), 867-880.

Weisz, N., Dohrmann, K., Elbert, T., 2007. Prog. Brain Res. 166, 61-70.

Wiersema, J., van der Meere, J., Roeyers, H., 2007. Developmental changes in error monitoring: an event-related potential study. Neuropsychologia 45 (8), 1649-1657

Yeung, N., Botvinick, M., Cohen, J., 2004. The neural basis of error detection: conflict monitoring and the error-related negativity. Psychol. Rev. 111 (4), 931-959. 